\title{
Ultimate Performance of the ATLAS Superconducting Solenoid
}

Roger Ruber, Yasuhiro Makida, Masanori Kawai, Yoshinari Kondo, Yoshikuni Doi, Tomiyoshi Haruyama, Friedrich Haug, Herman ten Kate, Taka Kondo, Olivier Pirotte, Jos Metselaar, Shoichi Mizumaki, Gert Olesen, Edo Sbrissa, and Akira Yamamoto

\begin{abstract}
A 2 tesla, 7730 ampere, 39 MJ, 45 mm thin superconducting solenoid with a 2.3 meters warm bore and 5.3 meters length, is installed in the center of the ATLAS detector and successfully commissioned. The solenoid shares its cryostat with one of the detector's calorimeters and provides the magnetic field required for the inner detectors to accurately track collision products from the LHC at CERN. After several years of a stepwise construction and test program, the solenoid integration $100 \mathrm{me}$ ters underground in the ATLAS cavern is completed. Following the on-surface acceptance test, the solenoid is now operated with its final cryogenic, powering and control system. A re-validation of all essential operating parameters is completed. The performance and test results of underground operation are reported and compared to those previously measured.
\end{abstract}

Index Terms-Detector, magnet, performance.

\section{INTRODUCTION}

$\mathbf{T}$ HE ATLAS superconducting solenoid sits in the center of the detector set-up providing the magnetic field crucial for the momentum measurement of charged particle tracks in the inner detector. The detector set-up consists furthermore of an electromagnetic calorimeter, hadron calorimeter and muon spectrometer. Due to the central location of the superconducting solenoid it is of extreme importance that it does not disturb the energy measurement of particles by the calorimeters located further outwards. A very convenient solution was found in this thin superconducting solenoid with indirect cooling. To minimize the overall wall thickness and enhance the transparency for particles the solenoid shares the required cryostat with the electromagnetic calorimeter. Being filled with liquid argon it doubles as thermal shield for the solenoid.

The solenoid has a stored energy of $39 \mathrm{MJ}$, a cold mass of 5.6 tons and a thickness of $45 \mathrm{~mm}$ equivalent to $0.66 X_{0}$ (radiation length). For the coil to be this thin, it is wound with a single layer of specially developed high-strength aluminum stabilized $\mathrm{NbTi}$ superconductor [1]. The solenoid coil is mounted on the inner warm vessel of the Barrel Cryostat by 23 triangular glass

Manuscript received August 28, 2006

R. J. M. Y. Ruber, F. Haug, H. H. J. ten Kate, O. Pirotte, G. Olesen, and E. Sbrissa are with CERN, CH-1211 Geneva 23, Switzerland (e-mail: ruber@cern. ch).

Y. Makida, M. Kawai, Y. Kondo, Y. Doi, T. Haruyama, T. Kondo, and A. Yamamoto are with KEK, Tsukuba, 305-0801 Japan.

S. Mizumaki is with Toshiba Co., Tsurumi, Yokohama, 230-0045 Japan.

Color versions of one or more of the figures in this paper are available online at http://ieeexplore.ieee.org.

Digital Object Identifier 10.1109/TASC.2007.899022
TABLE I

MAIN PARAMETERS OF THE ATLAS SOLENOID

\begin{tabular}{ll}
\hline \hline Central Field & $2.0 \mathrm{~T}$ \\
Operating Current & $7730 \mathrm{~A}$ \\
Coil Size & $5.3 \mathrm{~m}(\mathrm{l}) \times 2.4 \mathrm{~m}(\Phi) \times 45 \mathrm{~mm}(\mathrm{t})$ \\
Warm Bore Diamter & $2.3 \mathrm{~m}$ \\
Wall Thickness & $0.66 \mathrm{X}_{0}$ \\
Stored Energy & $39 \mathrm{MJ}$ \\
Cold Mass & $5.6 \mathrm{tonnes}$ \\
Inductance & $1.35 \mathrm{H}$ \\
Conductor Size & $30 \times 4.25 \mathrm{~mm}^{2}$ \\
Conductor Area Ratio & $1: 0.9: 14(\mathrm{NbTi}: \mathrm{Cu}: \mathrm{Al})$ \\
Number of Strands & 12 \\
Strand Diameter & $1.25 \mathrm{~mm}$ \\
$\mathrm{I}_{C}$ at $5 \mathrm{~T}, 4.2 \mathrm{~K}$ & $22 \mathrm{kA}$ \\
Conductor RRR & $500-570$ \\
Cooling Mode & 2 -phase forced flow or thermo-syphon \\
\hline \hline
\end{tabular}

fiber supports [2] with the services connection side (chimney) fixed while the opposite side can slide in axial direction [3]. The main parameters of the solenoid are listed in Table I. Its major features are described elsewhere [4].

\section{INSTALLATION}

During the last year the superconducting solenoid has been installed in the ATLAS experimental cavern, $100 \mathrm{~m}$ underground. The lowering into the cavern is shown in Fig. 1. After arrival in the cavern it has been encapsulated by the hadron calorimeter which also serves as a return yoke for the magnetic flux. A $6 \mathrm{~m}$ high chimney connects the solenoid cooling and superconducting bus-lines to a services cryostat, the control dewar, on top of the detector set-up. A general layout of the installation is shown in Fig. 2 while Fig. 3 shows the internal layout of the Barrel Cryostat.

During the underground installation two joints had to be made. One joint of the superconducting bus-lines, cryogenics cooling lines and instrumentation signal cabling is located between the Barrel Cryostat and chimney. And a second joint between the chimney and control dewar. This joint also includes a bellows to allow a $20 \mathrm{~mm}$ movement in any direction in between Barrel Cryostat and control dewar in case of an earthquake.

The solenoid was tested twice before the installation and commissioning: at the factory site in Japan [5] and at the surface integration hall in CERN [6], [7]. These tests were always performed in an air-core environment with the chimney in horizontal position. The commissioning was the first operation with the iron yoke from the hadron calorimeter and the chimney 


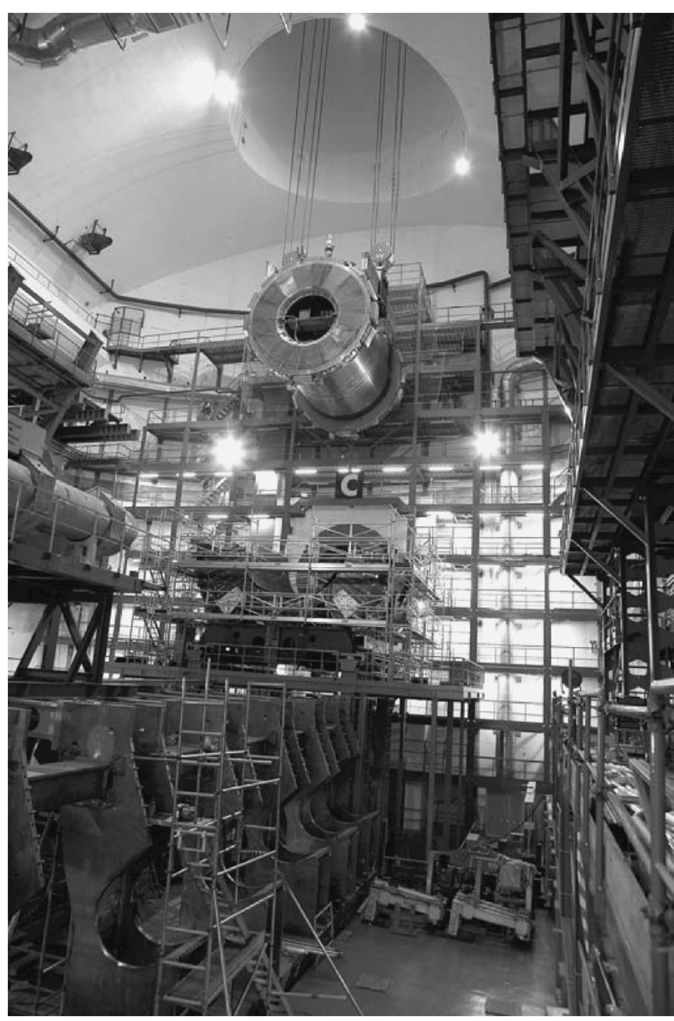

Fig. 1. Descent of the Barrel Cryostat with solenoid and calorimeter into the underground cavern.

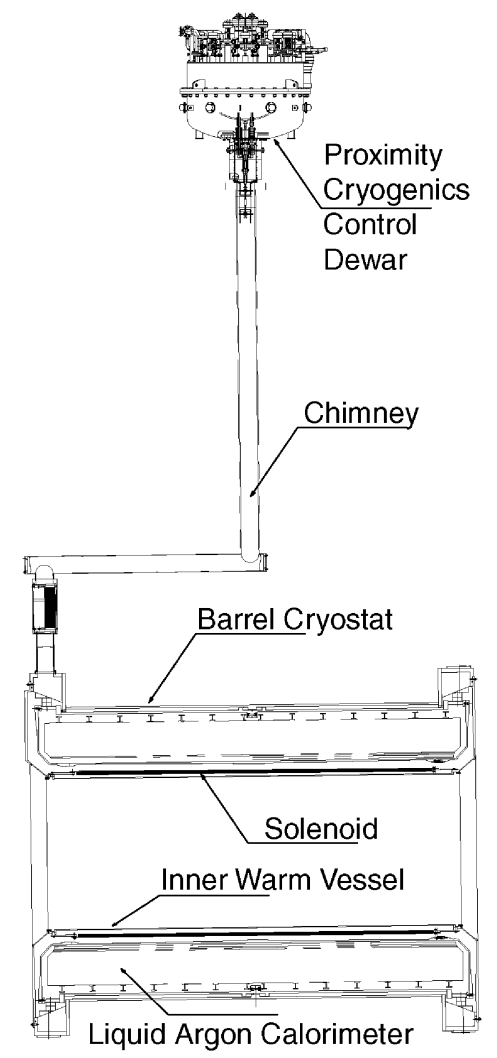

Fig. 2. General layout of the installation in the detector set-up.

vertical. It was also a first with the final cryogenic, powering and control systems.

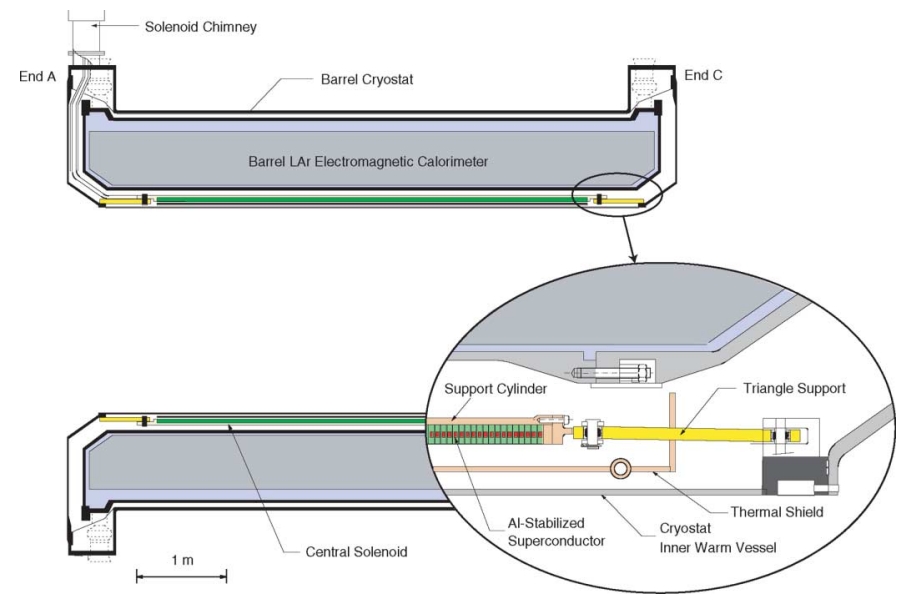

Fig. 3. Cross-section of the Barrel Cryostat showing the layout of the superconducting solenoid and the liquid argon calorimeter.

\section{INSTRUMENTATION AND PROTECTION}

The solenoid is equipped with more than two hundred sensors: voltage taps, thermometers, strain gauges, position meters, superconducting quench detectors and pick-up coils. Furthermore seven quench protection heaters (QPHTs) are installed near the conductor. Five of these QPHTs are included in a quench detector and protection unit, the Magnet Safety System (MSS). This unit applies a $1.0 \mathrm{~s}$ window in which an input signal must be above threshold before releasing a quench detection trigger initiating a so called Fast Dump. The Fast Dump will isolate the solenoid from the power converter and fire the QPHTs to create additional normal zones and spread the energy more uniformly over the coil. 72 High purity aluminum quench propagation strips (RRR $\approx 3000$ ), thickness $1 \mathrm{~mm}$ and width $100 \mathrm{~mm}$, are glued to the inner surface of the coil windings to enhance the turn-to-turn normal zone propagation velocity.

In case of e.g. a cooling or power failure the solenoid is discharged through an external dump unit consisting of diodes and resistors. This is a so called Slow Dump, initiated by the MSS it also isolates the solenoid from the power converter. More over it will also modify the settings of the proximity cryogenics system to change over from 2-phase forced flow cooling to thermosyphon cooling. The thermo-syphon cooling is based on a 250 liter liquid helium reservoir situated in the Control Dewar at the end of the chimney.

\section{COMMISSIONING}

\section{A. Field}

In the ATLAS detector, where the solenoid is surrounded by the hadron calorimeter, the nominal operational current to reach the desired 2.0 T field strength in the center is $7730 \mathrm{~A}$. During the previous (air core) testing campaigns, a current of $8000 \mathrm{~A}$ was required to reach the $2.0 \mathrm{~T}$ field, indicating that the iron of the hadron calorimeter contributes with $3.5 \%$ to the field strength. During the commissioning a maximum current of 7995 A was applied, see Fig. 4. At this current the MSS automatically 


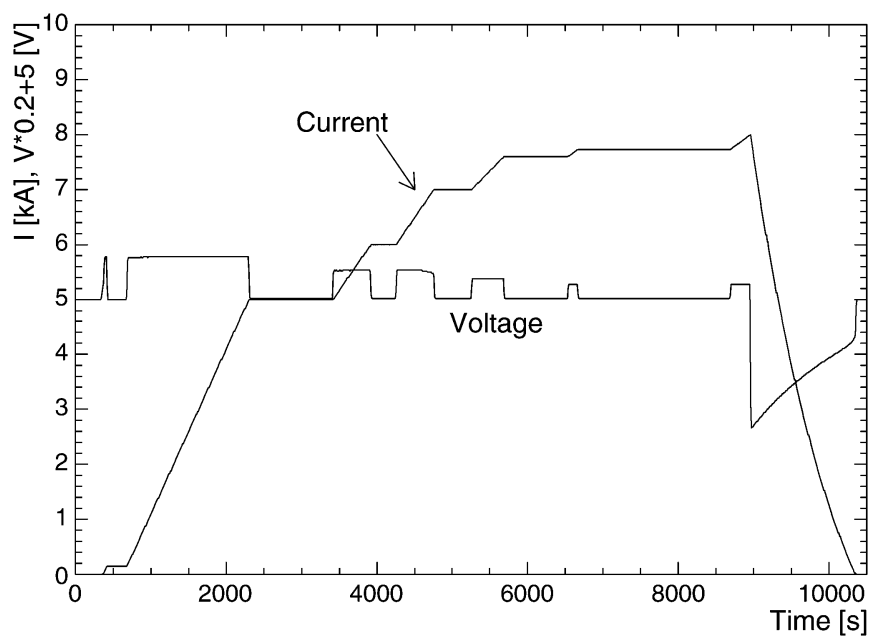

Fig. 4. Current and voltage during an excitation to $8 \mathrm{kA}$ with subsequent Slow Dump. The excitation is in steps of 5, 6, 7, 7.6 and $7.73 \mathrm{kA}$.

initiated a Slow Dump as it is the programmed over-current setting.

During the present commissioning process, the solenoid current was brought up 8 times to the nominal value $(7730 \mathrm{~A})$ from $0 \mathrm{~A}$. The reproducibility of the field strength measured with NMR probes located around the inner warm vessel of the cryostat was in the order of $\pm 0.1 \times 10^{-4} \mathrm{~T}$, indicating no significant hysteresis effect of the iron.

\section{B. Cooling and Cryogenic System}

The ATLAS superconducting solenoid is cooled to baseline temperature conditions with two helium refrigerators which are shared with the superconducting toroids. One refrigerator is used for cool down to $75 \mathrm{~K}$ after which the second refrigerator takes over and keeps the cold mass at $4 \mathrm{~K}$. The first refrigerator is then used to cool the thermal shields. The cool down time is approximately 4 days for the first phase and one day for the second [8].

During normal operation a forced two-phase cooling flow is used. In case of a quench or refrigerator failure, the cooling mode is switched automatically to thermo-syphon cooling. The solenoid goes into Slow Dump, which takes less than 20 minutes, while the available time span for thermo-syphon operation with the liquid helium available in the reservoir of the control dewar was demonstrated to extend to over one hour.

\section{Quench Protection and Hotspot Temperature}

During the factory tests there were two unexpected quenches, at 7016 and $7546 \mathrm{~A}$. A maximum current of $8.4 \mathrm{kA}$ was reached. After transport to CERN and installation in the Barrel Cryostat there were two re-training quenches at 7946 and $8112 \mathrm{~A}$. The maximum current was limited by the power system to $8.2 \mathrm{kA}$. No re-training was observed during the commissioning.

As part of the commissioning program, a heater initiated quench and Fast Dumps were provoked at several currents. The heater initiated quench was provoked with one of the Quench Protection Heaters located at the center of the coil at a current of $6000 \mathrm{~A}$, after which the MSS fires the remaining QPHTs. A maximum coil temperature of $84 \mathrm{~K}$ was measured. This is

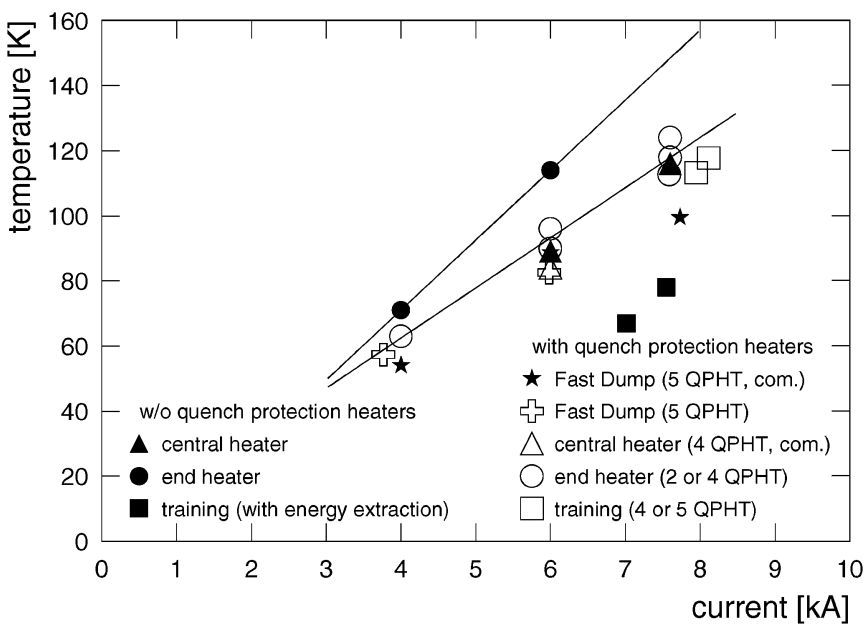

Fig. 5. Hot spot temperature as function of the coil current with and without the quench protection heaters (QPHTs) activated and for different quench ignition points. Energy extraction indicates usage of a different external protection system [5]. The commissioning data is indicated by "com". Note that the Fast Dump and central heater induced quenches at $6 \mathrm{kA}$ give the same hotspot temperature.

only slightly below an $89 \mathrm{~K}$ measured during a similar induced quench during a previous test in which the remaining QPHTs were not fired. See Fig. 5. It is however clear from the data presented in this figure that if the quench is initiated at the edge (end) of the coil there is a significant hotspot temperature difference between the situation were the QPHTs are fired or not. The straight lines in Fig. 5 are fitted through the hotspot temperature data for the end heater induced quenches were the QPHTs fired or not respectively.

Three Fast Dumps were provoked at 4000, 6000 and 7730 A. At the nominal current of 7730 A the hotspot temperature reaches just $100 \mathrm{~K}$. The maximum pressure observed in the cryogenic cooling lines of the coil was 7.3 bar. When a quench is detected, the solenoid cooling lines are immediately isolated from the proximity cryogenic system and the pressure is released through two dedicated lines (at the inlet and return).

The signals of two superconducting quench detectors (SQD) [9] installed along the length of the coil and the chimney are shown in Fig. 6. It can be seen that it takes slightly longer for the complete chimney to quench than the coil. In previous tests the chimney was always in horizontal position. It was then observed that the signal of the SQD in the chimney would decrease during several seconds after the coil SQD had reached its maximum level [6]. This behavior is no longer observed with the chimney in its final vertical position. It is believed to be due to recooling of the chimney by cold gas returning from the coil and possibly trapped in an S-curved extension of the chimney used for the test in horizontal position.

From Fig. 6 it can be seen that after a Fast Dump initiation the delay between the start of the normal zone in the coil and the chimney is in the order of a second only. The QPHTs are all located on the coil, there are none in the chimney.

\section{Mechanical Behavior}

The coil position is monitored by potentiometers mounted in axial and circumferential direction at both ends of the coil. At 


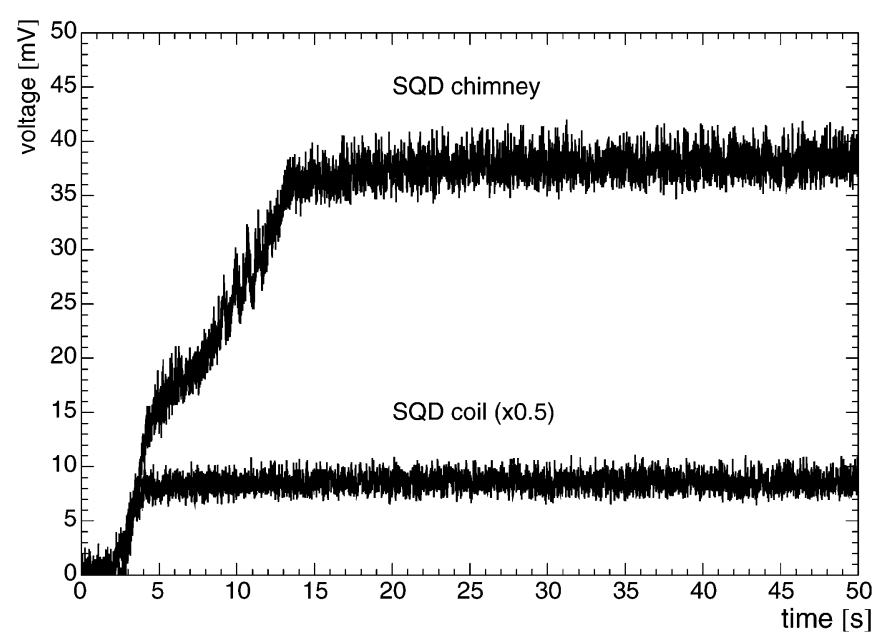

Fig. 6. SQD signal evolution for the coil and the chimney during a quench. The Fast Dump from 7730 A starts at $t=0 \mathrm{~s}$.

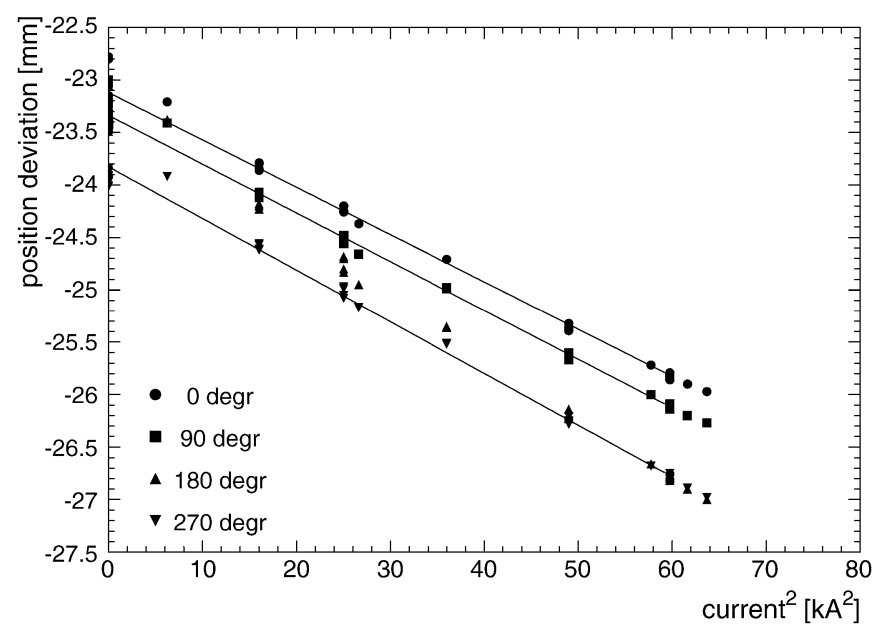

Fig. 7. Axial deformation during excitation at different locations around the circumference of the coil. Measurement accuracy $\pm 0.5 \mathrm{mmm}$, reproducibility $\leq 0.1 \mathrm{~mm}$.

the "fixed" end (chimney, "end A") movement is limited to $\approx 0.5$ $\mathrm{mm}$. During cooldown the average of the measured contraction is $-23.3 \pm 0.5 \mathrm{~mm}$ at the sliding end and $-0.2 \pm 0.5 \mathrm{~mm}$ at the "fixed" end, which corresponds to $0.41 \%$ of the $5698.2 \pm 0.8$ $\mathrm{mm}$ coil length. After excitation to $7730 \mathrm{~A}$ the contraction becomes $-25.1 \pm 0.5 \mathrm{~mm}$ and $-1.3 \pm 0.5 \mathrm{~mm}$ respectively, similar as measured during the on-surface test [6]. The reproducibility of the measured position during excitation is $\leq 0.1 \mathrm{~mm}$. Fig. 7 shows the sum of the axial contraction as measured at each of the four measurement locations around the circumference of the coil. The different offsets at $0 \mathrm{~A}$ are due to differences during cool down. As the installation, aligned at room temperature, was intentionally $13.2 \pm 1.1$ offset from the center the final position of the coil after cool down and excitation is $0.0 \pm 1.6 \mathrm{~mm}$. This being the center of the detector with respect to the interaction point.

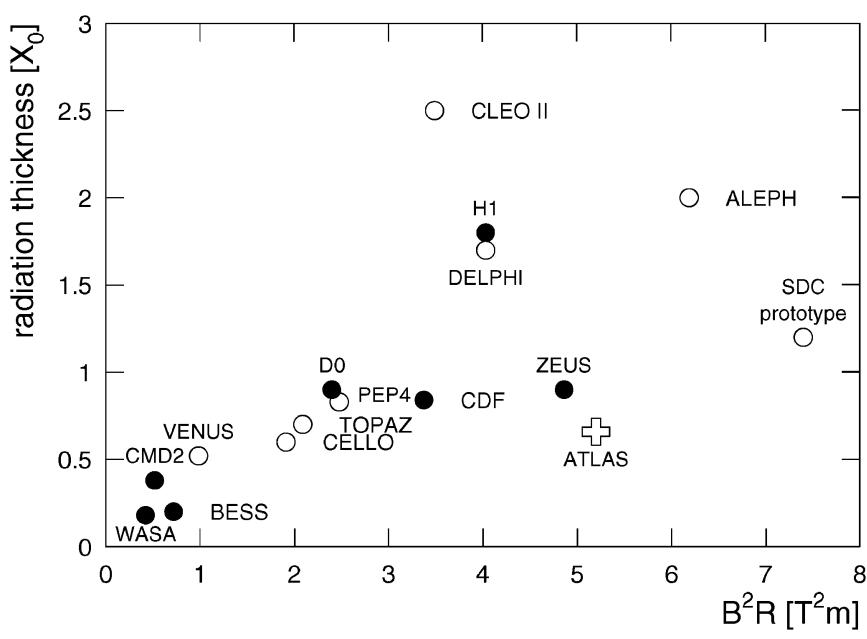

Fig. 8. The thickness of the ATLAS solenoid, in radiation lengths, compared to solenoids from other detector systems as a function of $B^{2} \cdot R$. Solid circles indicate detector systems presently under operation.

\section{CONCLUSIONS}

The ATLAS superconducting solenoid is successfully commissioned. Safe operation has been confirmed up to the maximal current allowed by the powering and safety systems. The combination of quench detection system, quench protection heaters and thermo-syphon cooling guarantee an excellent protection against an unexpected quench or failure of the external services. Due to its efficient design the solenoid combines a high particle transparency with a high bending power as is demonstrated in Fig. 8. The solenoid is now ready for the start of the experimental data taking.

\section{ACKNOWLEDGMENT}

The authors like to thank their colleagues for their extensive efforts during the integration and test. This research is supported by the ATLAS Collaboration and in particular by KEK in Japan and CERN in Switzerland.

\section{REFERENCES}

[1] K. Wada et al., "Development of high-strength and high-RRR aluminium-stabilized superconductor for the ATLAS thin solenoid," IEEE Trans. Appl. Superconduct., vol. 10, p. 373, 2000.

[2] A. Yamamoto et al., "Developments towards ultra-thin superconducting solenoid magnets for high energy particle detectors," Nucl. Phys., vol. B78, p. 565, 1999.

[3] S. Mizumaki et al., "Fabrication and mechanical performance of the ATLAS central solenoid," IEEE Trans. Appl. Superconduct., vol. 12, p. 416, 2002.

[4] A. Yamamoto et al., "Design and development of the ATLAS central solenoid magnet," IEEE Trans. Applied Superconduct., vol. 9, p. 852, 1999.

[5] Y. Makida et al., "Quench protection and safety of the ATLAS central solenoid," IEEE Trans. Appl. Superconduct., vol. 12, p. 407, 2002.

[6] R. J. M. Y. Ruber et al., "ATLAS superconducting solenoid on-surface test," IEEE Trans. Appl. Superconduct., vol. 15, p. 1283, 2005.

[7] Y. Doi et al., Final Testing of the ATLAS Central Solenoid before Installation. Beijing, China:, 2004.

[8] F. Haug et al., Commissioning of the ATLAS Central Solenoid Cryogenics. Prague, Czech Republic: , 2006.

[9] A. Dudarev et al., "Quench propagation and detection in the superconducting bus-bars of the ATLAS magnets," IEEE Trans. Appl. Superconduct., vol. 10, p. 381, 2000. 